\title{
Patellar Tendon Reflex and Vastus Medialis Hoffmann Reflex Are Down Regulated and Correlated in Women With Patellofemoral Pain
}

\author{
Marcella Ferraz Pazzinatto, MS, ${ }^{a, b}$ Danilo de Oliveira Silva, MS, ${ }^{a, b}$ \\ Amanda Schenatto Ferreira, MS, ${ }^{a}$ Marina Cabral Waiteman, MS, ${ }^{a}$ Evangelos Pappas, PhD, ${ }^{\mathrm{C}}$ \\ Fernando Henrique Magalhães, $\mathrm{PhD},{ }^{d}$ Fábio Mícolis de Azevedo, $\mathrm{PhD}^{\mathrm{a}}$ \\ From the a Physical Therapy Department, School of Science and Technology, University of São Paulo State (UNESP), Presidente Prudente, \\ Brazil; ${ }^{b}$ La Trobe Sports and Exercise Medicine Research Centre (LASEM), School of Allied Health, La Trobe University, Bundoora, Australia; \\ ${ }^{c}$ Faculty of Health Sciences, Discipline of Physiotherapy, University of Sydney, Sydney, Australia; and ${ }^{d}$ School of Arts, Sciences, and \\ Humanities, University of Sao Paulo, Sao Paulo, Brazil.
}

\begin{abstract} and pain-free controls.

Design: Cross-sectional observational study.

Setting: Laboratory of biomechanics and motor control. T-reflex (elicited via mechanical percussion on the patellar tendon) were estimated. control group $(r=0.72 ; P<.001)$. impaired excitability of the stretch reflex pathway associated with PFP.

Archives of Physical Medicine and Rehabilitation 2019;100:514-9

(c) 2018 by the American Congress of Rehabilitation Medicine
\end{abstract}

Objectives: The aims of this study were threefold: (1) to compare the amplitude of patellar tendon reflex (T-reflex) between women with patellofemoral pain (PFP) and pain-free controls; (2) to compare the amplitude of vastus medialis Hoffmann reflex (VM H-reflex) between women with PFP and pain-free controls; (3) to investigate the association between the amplitude of patellar T-reflex and VM H-reflex in women with PFP

Participants: Thirty women with PFP and 30 pain-free women aged 18 to 35 years $(\mathrm{N}=60)$.

Main Outcome Measures: Peak-to-peak amplitudes of maximal VM H-reflex (elicited via electrical stimulation on the femoral nerve) and patellar

Results: Women with PFP had significant lower amplitude of patellar T-reflex (mean difference $=0.086$; $95 \%$ confidence interval $=0.020$ to $0.151 ; P=.010$; moderate effect) and VM H-reflex (mean difference $=0.150 ; 95 \%$ confidence interval $=0.073$ to $0.227 ; P<.001 ;$ large effect) compared to pain-free controls. The VM H-reflex was strongly correlated with patellar T-reflex in both PFP group $(r=0.66 ; P<.001)$ and

Conclusions: As the T-reflex is easier to perform than H-reflex assessments in a clinical setting, it represents a feasible option to assess the
Supported by the Sao Paulo Research Foundation - FAPESP (grant no. 2016/50320-0) and (scholarship no. 2014/10839-0, 2015/10631-3, and 2016/19784-0). The financial sponsor played no role in the design, execution, analysis and interpretation of data, or writing of the study. variety of proximal (hip and trunk), local (knee), and distal (ankle and foot) biomechanical factors have been proposed to explain the pathomechanisms underlying PFP. ${ }^{5,6}$ However, when inserted in regression models, previously reported biomechanical factors do not explain high percentages of pain and/or functional deficits in individuals with PFP, highlighting the multifactorial nature and poor understanding of the condition. ${ }^{7,8}$

The Hoffmann reflex (H-reflex), which is considered an "electrical homologous" of the stretch reflex, is elicited by a transcutaneous electrical stimulus that activates afferent fibers 
from muscle spindles (which connects directly to spinal motoneurons). ${ }^{9}$ This might lead to the generation of a compound muscle action potential that is recorded with surface EMG electrodes at the associated muscle, termed H-reflex. ${ }^{10}$ This technique has been widely used to assess the excitability of the stretch reflex pathway and hence to investigate a variety of spinal cord mechanisms that play important roles in movement control. ${ }^{9}$ In this direction, a decreased excitability of the quadriceps H-reflex has been shown in response to nociceptive stimulus (saline injection) ${ }^{11}$ and even in response to anticipation of pain (ie, stimuli associated with pain but without nociceptive discharge), ${ }^{12}$ which suggests that pain may contribute to the down regulation of the H-reflex in some musculoskeletal conditions as PFP. ${ }^{13}$ Accordingly, De Oliveira Silva et $\mathrm{al}^{13}$ reported that the amplitude of vastus medialis (VM) H-reflex is lower in women with PFP compared to pain-free controls. Moreover, in a subsequent study, the lower amplitude of VM H-reflex in women with PFP was associated with higher pain levels, lower self-reported physical function, and greater chronicity of pain. ${ }^{14}$ A plausible hypothesis, yet to be tested by means of prospective studies, is whether the impaired VM H-reflex excitability is a risk factor for PFP or, alternatively, a byproduct of long-term pain. ${ }^{15}$ Such an investigation might help in the early identification of PFP and/or in the design of more effective interventions.

Despite the promising contribution of neurophysiological factors to the understanding of the underlying mechanisms associated with PFP, VM H-reflex evaluation may not be feasible in the clinical setting as it requires a rigorous control of the electrical stimulation to be delivered to the mixed muscle nerve. Alternatively, the stretch reflex pathway can also be assessed by a mechanical (tendon percussion) stimulus, also called tendon jerk or tendon reflex (T-reflex). Despite some differences such different sensitivity to conditioning stimuli and dependence from fusimotor drive, ${ }^{16}$ the $\mathrm{H}$ - and T-reflexes can be interpreted similarly from a clinical point of view. Both reflect the excitability of the stretch reflex pathway by means of electrical (H-reflex) or mechanical (T-reflex) stimulation of Ia afferent fibers that impinge directly onto alpha motoneurons. ${ }^{9}$ Furthermore, for muscles in which an H-reflex is not easily obtained, T-reflex is considered an alternative method for assessing motoneuron excitability. ${ }^{17}$ The T-reflex is routinely used in clinical assessments of spinal cord integrity, since a basic mechanical percussion only requires a simple clinical hammer, which is easier and inexpensive as compared to electrical stimulation. ${ }^{18}$ Therefore, whether T-reflex is able to differentiate between individuals with and without PFP (as previously shown for H-reflex) is an important, clinically-relevant research question, that remains unanswered. If patellar T-reflex of women with PFP present similar results as VM H-reflex in differentiating woman with and without PFP, it may be proven as a promising tool to be

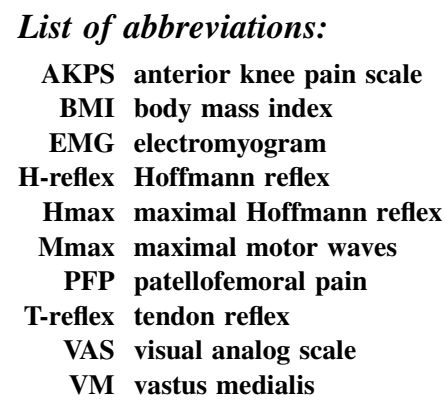

used in the clinical settings, thereby increasing the clinical applicability of assessing the excitability of spinal pathways in individuals with PFP.

In this context, the aims of this study were threefold: (1) to compare the amplitude of patellar tendon reflex (T-reflex) between women with patellofemoral pain (PFP) and pain-free controls; (2) to compare the amplitude of vastus medialis Hoffmann reflex (VM H-reflex) between women with PFP and pain-free controls; (3) to investigate the association between the amplitude of patellar T-reflex and VM H-reflex in women with PFP and pain-free controls. Based on previous research on the H-reflex, ${ }^{13}$ it was hypothesized that the amplitude of both T-reflex and VM H-reflex would be lower in women with PFP as compared to pain-free controls. It was also hypothesized that there would be significant correlations between T- and H-reflex.

\section{Methods}

\section{Participants}

For this cross-sectional observational study, 30 women with PFP and 30 pain-free women aged 18 to 35 years were recruited by opportunity sampling between September 2016 and May 2017 via advertisements in fitness centers, public places for physical activity and social media. The study was approved by the local human ethics committee, and all participants provided written informed consent.

The eligibility criteria were based in the last PFP consensus statement and previous studies. ${ }^{14,19-21}$ The following inclusion criteria for PFP participants were assessed by a clinician $(>5 y$ experience) (1) presence of anterior knee pain when performing at least 2 of the following activities: sitting for prolonged time, squatting, kneeling, running, ascending and descending stairs, jumping and landing; (2) insidious onset symptoms lasting at least 1 month; (3) worst pain level in the previous month corresponding to at least 30 millimeters in the visual analog pain scale (VAS); (4) unilateral PFP. Women were included in the pain-free group if they had no signs or symptoms of PFP or other neurological or musculoskeletal condition. Exclusion criteria, also assessed by a clinician, for both PFP and pain-free groups included signs or symptoms of any other knee dysfunction (eg, meniscal injury, ligament tears, knee osteoarthritis, Osgood-schlatter, patellar tendinopathy) history of surgery in any lower limb joint, history of patellar subluxation, and referred pain coming from the lumbar spine. Those who had received oral steroids, opiate treatment, acupuncture, physiotherapy, or any other treatment for pain during the preceding 6 months were also excluded from this study. Participants were asked to not take any medication or practice unusual types of physical activity in the previous day of the data collection.

\section{Characteristics of the participants}

Demographic data were collected prior to testing, including age, weight, height, body mass index (BMI), and subcutaneous adipose thickness of the thigh. A skinfold caliper was used to measure the subcutaneous adipose thickness. ${ }^{22}$ All participants were asked to rate their worst knee pain intensity in the last month and knee pain before the data collection on VAS (0-100 mm). Additionally, duration of knee-related symptoms (months) and self-reported 
Table 1 Characteristics of the participants

\begin{tabular}{lrr}
\hline Characteristic & Pain-Free Controls & PFP Group \\
\hline Age (y) & $22.47 \pm 3.19$ & $21.83 \pm 3.35$ \\
Body mass (kg) & $58.10 \pm 7.07$ & $62.00 \pm 7.98$ \\
Height (m) & $1.62 \pm 0.06$ & $1.62 \pm 0.06$ \\
BMI & $22.05 \pm 2.63$ & $23.60 \pm 3.39$ \\
Subcutaneous adipose thickness of the thigh (mm) & $11.56 \pm 4.42$ & $13.30 \pm 3.81$ \\
Function (AKPS) & $97.70 \pm 6.27$ & $70.30 \pm 11.84^{*}$ \\
Symptoms duration (mo) & $\mathrm{NA}$ & $52.73 \pm 56.43$ \\
Worst knee pain in the last month (VAS) & $\mathrm{NA}$ & $54.10 \pm 16.95$ \\
Knee pain before the data collection (VAS) & $\mathrm{NA}$ & $16.14 \pm 12.00$ \\
\hline NOTE. The values are presented as mean \pm SD. & & \\
Abbreviation: NA, not applicable. & & \\
$\quad$ * Indicates $P<.05$. & &
\end{tabular}

knee function (anterior knee pain scale [AKPS]) were obtained. The AKPS has been validated for people with PFP and has been reported to demonstrate high test-retest reliability. ${ }^{23,24}$

\section{VM H-reflex and patellar T-reflex recording}

Bipolar surface electrodes, ${ }^{\text {a }}$ placed 2 centimeters distally to the motor point in the direction of the corresponding muscle belly (VM muscle), ${ }^{11,25}$ were used for recording H-reflex and patellar T-reflex. Electromyographic signals were amplified (final gain of 1000) using an MSC $1000 \mathrm{~V} 3$ system, $^{\mathrm{b}}$ with a common rejection mode ratio of $>130$ decibels, an input impedance of $1000 \Omega$, and a signal/noise ratio of $<3 \mathrm{mV}$. ${ }^{13,14}$ Signals were sent to an analog to digital interface ${ }^{\mathrm{c}}$ with a 5 kilohertz sampling rate, and then stored for later offline processing. ${ }^{13,14} \mathrm{VM}$ H-reflex and patellar T-reflex were measured from symptomatic lower limb in PFP group and from dominant lower limb in control group. The reference electrode was placed over the contralateral medial malleolus to avoid movement artefact.

\section{VM H-reflex stimulation}

Direct motor waves and H-reflexes from VM were obtained by electrical stimulation ${ }^{\mathrm{d}}$ applied to the femoral nerve through surface electrodes (rectangular pulses, 1-ms duration). The selfadhesive cathode ${ }^{e}$ was placed on the skin over the femoral nerve in the inguinal canal (laterally to the femoral artery) and the anode $^{\mathrm{f}}$ on the gluteal fold. ${ }^{26}$ The intervals between consecutive stimuli ranged pseudorandomly from 10 to 15 seconds. These relatively large intervals were chosen to minimize afferent input from stretch receptors during repeated muscle stretch and to avoid depression of the H-reflex due to reduced neurotransmitter release (ie, homosynaptic depression).

\section{VM H-reflex assessment}

To record VM H-reflexes, the participants remained in the supine position, with the arms kept parallel to the trunk and the head resting in a neutral position. They were asked to relax completely and avoid making any voluntary effort during the trials. Initially, the H-reflex recruitment curve was obtained by progressively increasing the intensity of electrical stimulation in $0.5-$ to $1.0-\mathrm{mA}$ increments to find the largest observable H-reflex, as measured by the peak-to-peak amplitude of single unrectified electromyographic sweeps displayed by an oscilloscope. ${ }^{9,13,14}$ Based on the stimulation intensity found to evoke the maximal amplitude of H-reflex (Hmax), a sample of $10 \mathrm{Hmax}$ was obtained. After Hmax assessments, 4 maximal motor waves (Mmax) were evoked. ${ }^{13,14}$ As the amplitude of motor waves increases with the increase in stimulus intensity and then reaches a plateau, supraximal stimulus intensity was used to elicit Mmax. ${ }^{14,25}$

\section{Patellar T-reflex assessment}

Patellar T-reflex was elicited manually by a reflex hammer. The participants were seated with the legs dangling freely at $90^{\circ}$ of knee flexion. To maximize the amplitude and success rate of the reflex response, the most sensitive region that elicits the reflex was located and marked ${ }^{27}$ through 3 pretrials tendon taps performed at random time intervals (10 to $15 \mathrm{~s}$ ). The reflex response was allowed to stop naturally, and the next tap was applied only when the lower leg was stationary. A sample of 10 T-reflexes was obtained and a minimum rest period of 10 seconds was provided between taps. The neuromuscular responses to the tapping stimulus were evaluated by VM EMG measurement. The patellar T-reflex and the VM H-reflex assessment were performed randomly in the same day by the same rater. The randomization

Table 2 Comparison between women with PFP and pain-free controls

\begin{tabular}{lllll}
\hline Variables & Pain-Free Controls & PFP Group & Mean Difference (95\% CI) & ES \\
\hline VM H-reflex (\%Mmax) & $0.25 \pm 0.20$ & $0.10 \pm 0.08$ & $0.15(0.07 ; 0.22)^{*}$ & 0.97 \\
Patellar T-reflex (\%Mmax) & $0.23 \pm 0.16$ & $0.14 \pm 0.09$ & $0.09(0.02 ; 0.15)^{*}$ & 0.68 \\
\hline
\end{tabular}

NOTE. The values are expressed as mean \pm SD or as otherwise indicated.

Abbreviations: CI, confidence interval; ES, effect size.

* Indicates $P<.05$. 

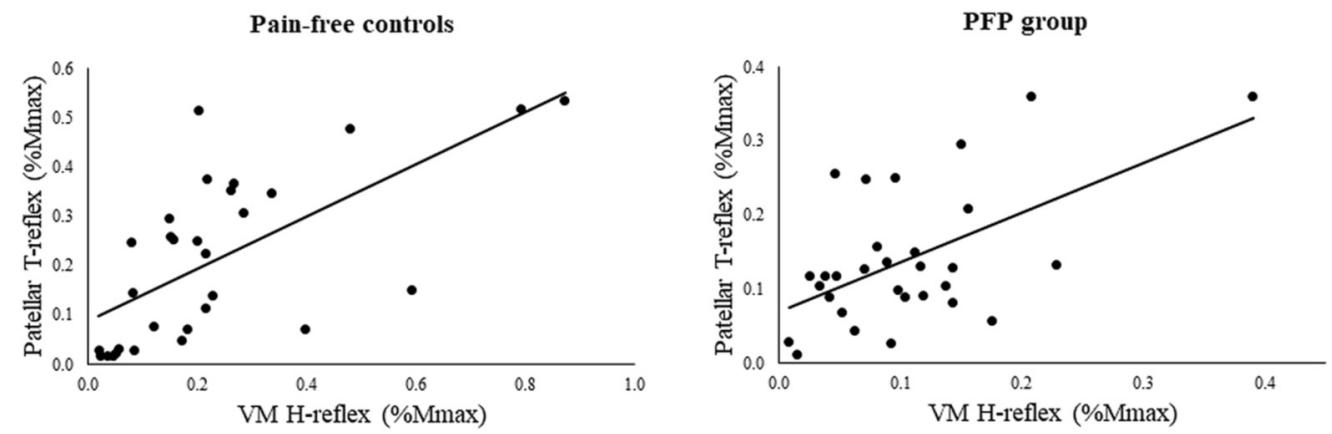

Fig 1 Scatterplots showing the correlation between the amplitude of VM H-reflex and patellar T-reflex in both PFP and control groups.

sequence was drawn up and kept off-site by a scientist not involved in data collection, using a random number generator with no stratification. Assessment order was concealed in sequentially numbered sealed opaque envelopes, which were opened by the rater at the moment of the data collection.

\section{Data processing}

Electromyographic signals were processed in MATLAB software. $^{g}$ The average Hmax was computed from the peak-to-peak amplitude of all 10 Hmax. Tmax was defined as the maximal peak-to-peak amplitude observed among 10 T-reflexes responses. Both Tmax and Hmax were normalized to the Mmax generated by supramaximal electrical stimulation.

\section{Statistical analysis}

Power calculation for this study was performed based on a previous study. ${ }^{13}$ Sample size was determined on the basis of predicted power to detect a difference of $0.14 \pm 0.12 \%$ Mmax in VM H-reflex between groups, considering a level of .05, 2-tailed and a power of $80 \%$. Based on the calculations performed with SamplePower software, a minimum sample size of 12 subjects per group was indicated.

Descriptive values (means and standard deviations) for each measurement were first obtained. All measurements were assessed for normality using the Shapiro-Wilk test. Mean age, weight, height, BMI, AKPS, VM H-reflex, and T-reflex measures were compared between groups using independent group $t$ tests. Effect sizes were calculated (Cohen's d) and the guidelines for interpreting the Cohen's d are as follows: 0 to 0.40 small effect, 0.41 to 0.70 moderate effect, 0.71 or higher large effect. ${ }^{28}$

A Pearson product-moment correlation matrix $(r)$ was used to explore the association between patellar T-reflex and VM H-reflex. Cohen has suggested the general guidelines of $r$ values 0.10 to 0.30 as small, 0.31 to 0.50 as moderate, and 0.51 to 1.00 as strong relations to aid in the interpretation of correlational findings. ${ }^{28}$ Statistical analyses were performed using Statistical Software for Social Sciences ${ }^{i}$ with an a priori level of significance of 0.05 .

\section{Results}

Women with PFP presented lower physical function $(P<.001)$ than pain-free controls, however groups presented similar anthropometric characteristics (table 1). The duration of kneerelated symptoms, worst knee pain in the last month and knee pain before data collection are also presented in table 1. The PFP group showed lower amplitude of VM H-reflex $(P<.001$; large effect) and patellar T-reflex ( $P=.010$; moderate effect) (table 2$)$. The VM H-reflex was strongly correlated with patellar T-reflex in both PFP group $(r=0.66 ; P<.001)$ and control group $(r=0.72$; $P<.001$ ) (fig 1).

\section{Discussion}

As hypothesized, the amplitude of patellar T-reflex (as assessed by VM EMG) and VM H-reflex were significantly lower in women with PFP as compared to pain-free controls. Additionally, the amplitude of patellar T-reflex was strongly correlated with the amplitude of VM H-reflex.

Lower VM H-reflex in women with PFP as compared to painfree controls has been previously reported, ${ }^{13}$ with the mean difference being quite similar between the previous and the present study. Interestingly, Park et $\mathrm{al}^{11}$ reported a $12 \%$ reduction of VM H-reflex in healthy individuals after immediately induced anterior knee pain (hypertonic saline injection). The findings of the present study showed that the VM H-reflex was $41.7 \%$ lower in women with PFP as compared to pain-free controls. The larger difference in our study may be explained by the presence of chronic pain in women with PFP. As reported recently, ${ }^{14}$ there is an association between chronicity and the excitability of VM H-reflex. These findings may suggest that early interventions aiming at increasing excitability of the monosynaptic stretch reflex pathway, such as cortico-motor facilitation via attention-oriented tasks or transcranial magnetic stimulation, ${ }^{29}$ proprioceptive exercises, ${ }^{30}$ sensory stimulation, ${ }^{31}$ or even reflex operant conditioning, ${ }^{32}$ may add to current evidence-based treatment for women with PFP. ${ }^{33}$ However, such interventions should be tested in randomized controlled trials before implementation in clinical practice.

Similar to VM H-reflex, the patellar T-reflex was lower in women with PFP as compared to pain-free controls. Despite some differences between $\mathrm{H}$ - and T-reflexes, both are mediated by the same spinal pathway, as the activation of Ia afferents elicits the excitatory drive that leads to the motoneuronal discharges, which might be assessed by means of either H- or T-reflexes. ${ }^{9}$ However, the H-reflex is generated by direct electrical stimulation of Ia afferents and hence bypasses muscle spindles, producing a single synchronous volley. ${ }^{9,25}$ Conversely, the T-reflex is elicited by a tap that stretches muscle spindles primary endings, thereby generating 
a more prolonged discharge in Ia afferents and hence also depends on the sensitivity to stretch of primary endings, a property that may be altered by the activity of $\gamma$ efferents. ${ }^{9}$ Moreover, $\mathrm{H}-$ and T-reflexes have been shown to behave differently as a function of a variety of condition stimuli. For instance, T-reflex is believed to be less sensitive to some conditioning protocols designed to assess the levels of presynaptic inhibition. ${ }^{16}$ Possibly, this could occur due to a post-tetanic potentiation of Ia terminals associated with the more prolonged burst of spikes. ${ }^{16}$ Therefore, as the specific mechanisms responsible for the impaired excitability of the stretch reflex in individuals with PFP are not understood, the question of whether altered T-reflex excitability would be observed in people with PFP could not be answered before the present study.

Taken together with the strong correlations that were found between VM H-reflex and patellar T-reflex, implementing the assessment of the stretch reflex excitability in individuals with PFP may be a good substitute for the H-reflex in the research setting as it is easier and cheaper. In addition, it could be also implemented in specialized clinics that have access to EMG. From a clinical perspective, it is important to evaluate the state of spinal neurophysiological pathways pre and postinterventions, given that regaining stretch reflex excitability might be a strategy (yet unexplored) to maintain the gains acquired during the rehabilitation programs. $^{14}$

\section{Study limitations}

Four participants of the PFP group had a dominant limb different from the control group. As are some biomechanical and neurophysiological differences between dominant and nondominant limbs that could have influenced the present results, further studies are needed to investigate potential neurophysiological differences between dominant and nondominant limbs in patients with PFP. In addition, some studies that assessed patellar T-reflex have built devices to firmly hold the reflex hammer at the desired position and automated the hammer so as to apply an equal force for each tendon tap. ${ }^{18,34}$ The method used in the present study may have involved some variation in the force that was applied among the tendon tap trials. However, we chose to keep the patellar T-reflex as clinical as possible, without any mechanical control of position and force in order to provide findings which are clinically applicable indeed.

\section{Conclusion}

This study provides a clinically feasible option (ie, the T-reflex) to assess the excitability of spinal cord pathways associated with PFP, without having to deal with the rigorous control of the electrical stimulation and the sophisticated apparatus involved in H-reflex assessment. Together with the positive strong correlations between VM H-reflex and patellar T-reflex, the present findings indicate that clinicians may consider using the patellar T-reflex to assess the excitability of the stretch reflex pathway in women with PFP in the clinical setting.

\section{Suppliers}

a. $\mathrm{Ag} / \mathrm{AgCl}$ electrodes; Medi-Trace.

b. MSC 1000 V3; LINX.

c. Model USB6009; National Instruments. d. Diapulsi 990; Quark Medical.

e. 1-cm diameter Valutrode; Axelgaard Manufacturing Co., LTD.

f. $5 \times 5 \mathrm{~cm}$ Valutrode; Axelgaard Manufacturing Co., LTD.

g. MATLAB; MathWorks Training Facility.

h. SamplePower; IBM.

i. SPSS, version 18.0; SPSS Inc.

\section{Keywords}

Knee; Inhibition; Motor neurons; Rehabilitation; Stretch reflex

\section{Corresponding author}

Marcella Ferraz Pazzinatto, Roberto Simonsen Street, 305, CEP 19060-900, Department of Physiotherapy FCT/UNESP, Presidente Prudente (SP), Brazil. E-mail address: marcellapazzinatto@gmail. com.

\section{References}

1. Boling M, Padua D, Marshall S, Guskiewicz K, Pyne S, Beutler A. Gender differences in the incidence and prevalence of patellofemoral pain syndrome. Scand J Med Sci Sports 2010;20:725-30.

2. Roush JR, Bay RC. Prevalence of anterior knee pain in 18-35 year-old females. Int J Sports Phys Ther 2012;7:396-401.

3. Crossley KM. Is patellofemoral osteoarthritis a common sequela of patellofemoral pain? Br J Sports Med 2014;48:409-10.

4. Wyndow N, Collins N, Vicenzino B, Tucker K, Crossley K. Is there a biomechanical link between patellofemoral pain and osteoarthritis? A narrative review. Sport Med 2016;46:1797-808.

5. Lankhorst NE, Bierma-Zeinstra SM, van Middelkoop M. Factors associated with patellofemoral pain syndrome: a systematic review. $\mathrm{Br}$ J Sports Med 2013;47:193-206.

6. Powers CM, Witvrouw E, Davis IS, Crossley KM. Evidence-based framework for a pathomechanical model of patellofemoral pain: 2017 patellofemoral pain consensus statement from the 4th International Patellofemoral Pain Research Retreat, Manchester, UK: part 3. Br J Sports Med 2017;51:1713-23.

7. Nakagawa TH, Serrão FV, Maciel CD, Powers CM. Hip and knee kinematics are associated with pain and self-reported functional status in males and females with patellofemoral pain. Int J Sports Med 2013; 34:997-1002.

8. de Oliveira Silva D, Briani R, Pazzinatto M, Ferrari D, Aragão F, de Azevedo F. Vertical ground reaction forces are associated with pain and self-reported functional status in recreational athletes with patellofemoral pain. J Appl Biomech 2015;31:409-14.

9. Pierrot-Deseilligny E, Burke D. The circuitry of the human spinal cord: its role in motor control and movement disorders. New York: Cambridge University Press; 2005.

10. Mezzarane RA, Elias LA, Magalhaes FH, Chaud VM, Kohn AF. Experimental and simulated EMG responses in the study of the human spinal cord. In: Turker H, editor. Electrodiagnosis in new frontiers of clinical research. Rijeka, Croatia: InTech; 2013. p 57-87.

11. Park J, Hopkins JT. Induced anterior knee pain immediately reduces involuntary and voluntary quadriceps activation. Clin J Sport Med 2013;23:19-24.

12. Tucker K, Larsson A-K, Oknelid S, Hodges P. Similar alteration of motor unit recruitment strategies during the anticipation and experience of pain. Pain 2012;153:636-43.

13. de Oliveira Silva D, Magalhães FH, Faria NC, et al. Lower amplitude of the Hoffmann reflex in women with patellofemoral pain: thinking beyond proximal, local, and distal factors. Arch Phys Med Rehabil 2016;97:1115-20. 
14. de Oliveira Silva D, Magalhães FH, Faria NC, et al. Vastus medialis H-reflex excitability is associated with pain level, self-reported function and chronicity in women with patellofemoral pain. Arch Phys Med Rehabil 2017:98:114-9.

15. Pazzinatto MF, de Oliveira Silva D, Pappas E, Magalhães FH, de Azevedo FM. Is quadriceps H-reflex excitability a risk factor for patellofemoral pain? Med Hypotheses 2017;108:124-7.

16. Mezzarane RA, Magalhães FH, Chaud VM, Elias LA, Kohn AF. Enhanced D1 and D2 inhibitions induced by low-frequency trains of conditioning stimuli: differential effects on $\mathrm{H}-$ and T-reflexes and possible mechanisms. PLoS One 2015;10:e121496.

17. McNeil CJ, Butler JE, Taylor JL, Gandevia SC. Testing the excitability of human motoneurons. Front Hum Neurosci 2013;7:152.

18. Dafkin C, Green A, Kerr S, Veliotes D, McKinon W. The accuracy of subjective clinical assessments of the patellar reflex. Muscle Nerve 2013;47:81-8

19. Crossley KM, Stefanik JJ, Selfe J, et al. 2016 Patellofemoral pain consensus statement from the 4th International Patellofemoral Pain Research Retreat, Manchester. Part 1: terminology, definitions, clinical examination, natural history, patellofemoral osteoarthritis and patientreported outcome measures. Br J Sports Med 2016;50:839-43.

20. Pazzinatto MF, de Oliveira Silva D, Pradela J, Coura MB, Barton C, de Azevedo FM. Local and widespread hyperalgesia in female runners with patellofemoral pain are influenced by running volume. J Sci Med Sport 2017;20:362-7.

21. Rathleff MS, Petersen KK, Arendt-Nielsen L, Thorborg K, GravenNielsen T. Impaired conditioned pain modulation in young female adults with long-standing patellofemoral pain: a single blinded crosssectional study. Pain Med 2016;17:980-8.

22. Selkow NM, Pietrosimone BG, Saliba SA. Subcutaneous thigh fat assessment: a comparison of skinfold calipers and ultrasound imaging. J Athl Train 2011;46:50-4.

23. Watson CJ, Propps M, Ratner J, Zeigler DL, Horton P, Smith SS. Reliability and responsiveness of the lower extremity functional scale and the anterior knee pain scale in patients with anterior knee pain. J Orthop Sport Phys Ther 2005;35:136-46.

24. Crossley KM, Bennell KL, Cowan SM, Green S. Analysis of outcome measures for persons with patellofemoral pain: which are reliable and valid? Arch Phys Med Rehabil 2004;85:815-22.

25. Palmieri RM, Ingersoll CD, Hoffman MA. The hoffmann reflex: methodologic considerations and applications for use in sports medicine and athletic training research. J Athl Train 2004;39:268-77.

26. Doguet V, Jubeau M. Reliability of H-reflex in vastus lateralis and vastus medialis muscles during passive and active isometric conditions. Eur J Appl Physiol 2014;114:2509-19.

27. Vickery HS, Smith PA. Surface electromyography reveals males have a slower patellar reflex than females. J Electromyogr Kinesiol 2012; 22:990-6.

28. Cohen J. Statistical Power Analysis for the Behavioral Sciences. 2nd ed. New York: Routledge Academic; 1988.

29. Master S, Tremblay F. Task-specific increase in corticomotor excitability during tactile discrimination. Exp Brain Res 2009;194: $163-72$.

30. Taube W, Kullmann N, Leukel C, Kurz O, Amtage F, Gollhofer A. Differential reflex adaptations following sensorimotor and strength training in young elite athletes. Int J Sports Med 2007;28:999-1005.

31. Perez MA, Field-Fote EC, Floeter MK. Patterned sensory stimulation induces plasticity in reciprocal ia inhibition in humans. J Neurosci 2003;23:2014-8.

32. Thompson AK, Wolpaw JR. The simplest motor skill: mechanisms and applications of reflex operant conditioning. Exerc Sport Sci Rev 2014;42:82-90.

33. Lack S, Neal B, De Oliveira Silva D, Barton C. How to manage patellofemoral pain-understanding the multifactorial nature and treatment options. Phys Ther Sport 2018;32:155-66.

34. Chandrasekhar A, Abu Osman NA, Tham LK, Lim KS, Wan Abas WA. Influence of age on patellar tendon reflex response. PLoS One 2013;8:e80799. 